\title{
Research on the Role of Matrix Metalloproteinase in the Patients with Stable Phase COPD Accompanied by Osteoporosis
}

\author{
Xinan Wang, " , Donghui Tian ${ }^{2}$, Jianfang Wang ${ }^{3}$, Xiuhe Ouyang ${ }^{1}$ \\ ${ }^{1}$ Department of Internal Respiratory, Binzhou People's Hospital, Binzhou, P. R. China \\ ${ }^{2}$ Department of Internal Neurology, Binzhou People's Hospital, Binzhou, P. R. China \\ ${ }^{3}$ Department of Public Health, Binzhou People's Hospital, Binzhou, P. R. China
}

Email address:

wangxinanshangdong@163.com (Xinan Wang)

${ }^{*}$ Corresponding author

\section{To cite this article:}

Xinan Wang, Donghui Tian, Jianfang Wang, Xiuhe Ouyang. Research on the Role of Matrix Metalloproteinase in the Patients with Stable Phase COPD Accompanied by Osteoporosis. American Journal of Clinical and Experimental Medicine. Vol. 5, No. 1, 2017 , pp. 19-25. doi: 10.11648/j.ajcem.20170501.15

Received: November 15, 2016; Accepted: January 20, 2017; Published: February 10, 2017

\begin{abstract}
Subheading: Role of Matrix Metalloproteinase in the Patients with Stable Phase COPD. Objective: The research aims to quantitatively evaluate the serum MMP-9, inhibitors of TIMP-1, TNF- $\alpha$, and OPG/RANK/RANKL in the patients with COPD accompanied by osteoporosis. Method: We selected 90 male patients with stable phase COPD who received treatment in our hospital from September 2012 to May 2014. They were divided into three groups by bone mineral density (BMD), COPD normal bone mass group, low bone mass group, and osteoporosis group of 30 patients each. MMP-9, TIMP-1, TNF- $\alpha$, and OPG/RANK/RANKL were detected with the ELISA method. The ELISA method was used to detect the levels of serum bone alkaline phosphatase (sBAP), serum osteocalcin (sOC), serum type I collagen cross-linked C telopeptide (sCTX). Result: There were significant differences in BMI and CAT scores between the osteoporosis group, the normal bone mass group, and the low bone mass group $(\mathrm{P}<0.01)$. The bone density of lumbar vertebra in the low bone mass group and the osteoporosis group decreased when compared with that in the normal bone mass group. The differences were statistically significant $(\mathrm{P}<0.05, \mathrm{P}<0.01)$. The density of femoral neck in the low bone mass group and the osteoporosis group was significantly lower than that in the normal bone mass group. And there were significant differences $(\mathrm{P}<0.01)$. There were significant differences in level of serum MMP-9 among the three groups $(\mathrm{P}<0.01)$. Conclusion: MMP-9, TNF- $\alpha$, and OPG/RANK/RANKL may play a collaborative role in occurrence and progression of pulmonary parenchyma damage and osteoporosis.
\end{abstract}

Keywords: Chronic Obstructive Pulmonary Disease, Bone Mineral Density, Osteoporosis, Matrix Metalloproteinase

\section{Introduction}

COPD has a high disability rate and case fatality rate, which are rising all the time. COPD may become the third most fatal disease by 2020 [1]. The existing research shows that COPD is a systemic disease involving multiple tissues and organs instead of a simple pulmonary disease. Osteoporosis (OP) is one the important systemic complications of COPD [2] and its morbidity in the COPD patients is significantly higher than that in the normal people. The patients with severe osteoporosis would suffer from decreasing vital capacity and total lung capacity with the increase of thoracic vertebrae fractures [3]. The pathogenesis of secondary osteoporosis of COPD still remains unclear. In the research by Duckers et al., more than $80 \%$ of the male COPD patients complicated by osteopenia or osteoporosis suffer from mild to moderate airflow obstruction [4]. Previous pathophysiologic research has shown that The pulmonary parenchyma damage and the bone structure damage are associated to some extent. The potential mechanism of such correlation still remains unclear [5]. It is inferred that it may be associated with the action of systemic inflammatory factors on both pulmonary parenchyma and 
bone matrix.

Matrix metalloproteinases (MMPs) are a set of endopeptidases containing zinc ions activated by calcium ions commonly found in various connective tissues, and essential enzymes during degradation of extracellular matrix (ECM) and basilar membrane. Research shows that the increase of MMP-9 will lead to increased degradation of ECM in the respiratory tract [6] and perforate the basilar membrane of the epithelial cells thus promoting emigration of the inflammatory cells and their aggregation in the target organ and further exacerbating the inflammatory responses. The tissue inhibitors metalloproteinase (TIMPs) are a set of endogenous glycoproteins that specifically inhibit the activity of MMPS [7]. TIMP-1 is primarily synthesized by macrophages, endothelial cells, fibroblasts etc. The MMP9/TIMP-1 ratio is currently considered to be an indicator for the dynamic balance between airway tissue damage and restoration. TIMP-1 blocks the activation of MMP-9 zymogen and inhibits the activity of the activated MMP-9 enzyme [8, 9]. TIMPs are also multifunctional molecules capable of inhibiting the activity of MMPs and playing a role similar to cell growth factors. They can promote the growth of proliferation fibroblasts and collagen synthesis, deposit ECM, and inhibit its degradation [10].

TNF- $\alpha$ is a multi-functional cytokine capable of inducing the endothelial cells to express adhesion molecules, mediating the leukocytes to adhere to the vascular endothelial cells, and causing aggregation of leukocytes at the inflammatory site [11]. In addition, it exhibits direct chemotaxis towards eosinophilic granulocytes. TNF- $\alpha$ can activate inflammatory leukocytes, particularly the macrophages and neutrophil and enables them to increase cytotoxicity and release more oxygen and nitrogen groups and other cytokines, such as IL-6, IL-8, and other inflammatory cellular chemotactic factors $[12,13]$. TNF- $\alpha$ can strengthen the extracellular proteolysis role of neutrophil and the strengthened proteolysis role is considered to be one of the mechanisms of emphysema development. Research finds that the level of sputum TNF- $\alpha$ increases significantly and the level of sputum TNF- $\alpha$ and the level of sputum IL-8 and the degree of airflow obstruction are significantly and positively correlated in the COPD patients [14]. It indicates that the increase of the level of TNF- $\alpha$ is essential to the development of COPD but it also indicates that the local expression of TNF-amay play a secondary role in an early stage of COPD.

OPG/RANKL/RANK cytokines regulation system is critical to osteoclast biology [15]. Some research points out that the change of this system is associated with the incidence of metabolic bone diseases. When there is a lack of estrogen, RANKL increases and OPG decreases thus leading to increased number and prolonged life of the mature osteoclasts. When the osteoblasts express and release RANKL, they are bond to the RANK on the osteoclastic precursor cytomembrane. The factors related to the tumor necrosis factor receptor are bound to the cytoplasmic region of RANK. TRAF-2, 5, and 6 can be bound to RANK. TRAF6 is associated with generation of osteoclasts. After TRAF6 and RANK are combined, the nuclear factor $-\kappa B$ is activated and transported into the nucleus. $N F-\kappa B$ increases the expression of c-Fos. c-Fos is further bond to the activated $\mathrm{T}$ nuclear factor for interaction thus initiating transcription of the production gene of osteoclasts [16-18] and finally inducing formation of mature osteoclasts.

Bone mineral density (BMD) refers to the bone mass of the unit volume (volume density) or the bone mass of the unit area (area density), which can measure the living organisms using noninvasive techniques. The bone mineral density reflects about $70 \%$ of the bone strength. The specific methods in clinical application include dual energy X-ray bone density determination method (DXA), peripheral dual energy X-ray bone density determination method (pDXA), quantitative computer tomography (QCT) etc. DXA measurement is a golden standard for diagnosing osteoporosis generally accepted in the international academic circle [19]. With the rapid development of cytobiology and molecular biology, research has found that the bone collagen metabolism is closely associated with bone metabolism. Excessive degradation of the bone collagen or decreased bone collagen synthesis would lead to declined bone elasticity and toughness, aggravated loss of bone mineral, and incidence of osteoporosis [20]. The trabecula of osteopenia bone and the damage to the fine structure of the osseous tissue cause the load pressure on the bone to decrease and easily cause bone fractures. These changes can be estimated on the basis of bone mineral density measured with a dual energy X-ray absorptiometry instrument. Measurement of BMD is one of the approaches for quantitative evaluation of osteoporosis. No clinical evaluation approach is available for bone quality and the bone metabolism markers are used to indirectly evaluate bone turnover.

In conclusion, it is inferred that MMP-9 and its inhibitor 1, the inflammatory factor $T N F-\alpha$, and the OPG/RANK/RANKL system may jointly participate and interact in osteoporosis secondary to COPD.

The research investigates the correlation between the serum MMP-9, its inhibitors -1, TNF- $\alpha$, and the OPG/RANK/RANKL system in the patients with chronic obstructive pulmonary disease complicated by (COPD), observes the correlation between MMP-9 and its inhibitors $1, \mathrm{TNF}-\alpha$ and bone turnover markers, and probes into the their roles in progression of COPD pulmonary functional damage and osteoporosis thus providing new possible directions and drug targets for early intervention treatment of COPD abnormal bone metabolism.

\section{Material and Method}

\subsection{Research Subjects}

We selected 90 male patients with stable phase COPD who received treatment in our hospital from September 2010 to May 2012. They were subjected to COPD diagnosis and 
severity grading according to the GLOD standard. They were divided into COPD normal bone mass group, low bone mass group, and osteoporosis group of 30 each based on the bone density results. Inclusion Criteria: The patients aged over 40 and diagnosed as having stable phase COPD with a previous smoking history (or ex-smokers). Exclusion Criteria: patients with severe cardiovascular and cerebrovascular diseases, hepatic failure and renal failure or disturbance of consciousness; patients definitely diagnosed as having other respiratory diseases such as asthma, bronchiectasia etc. in addition to COPD; patients with a history of skeleton system diseases such as bone fractures, osteomalacia etc.; patients with a history of rheumatic diseases, such as rheumatoid arthritis etc.; patients with endocrine metabolic diseases, such as hyperthyroidism, hyperparathyroidism etc.; the patients who received oral administration, inhalation, or intravenous injection of glucocorticoid within 3 months after being included into the group; the patients who received administration of drugs that influence skeleton metabolism within 1 year after being included into the group, including the patients who had been diagnosed as having osteoporosis and treated; patients with malignant tumors within 5 years after being included into the group. The general data and medical histories all of the patients included into the group were collected on the same day. The patients' quality of life was evaluated with the evaluation test of chronic obstructive pulmonary diseases. In the morning of the next day, $10 \mathrm{ml}$ of fasting elbow venous blood of each patient was drawn and used as sample. The research plan was approved by the Ethics Committee of the Affiliated Hospital of Inner Mongolia Medical University. All patients included into the groups signed an informed consent form.

\subsection{Somatometry and Determination of Pulmonary Functions}

The stature and body weight of each patient were recorded. The body mass index $(\mathrm{BMI})=$ body weight/stature $\left(\mathrm{kg} / \mathrm{m}^{2}\right)$ was computed with a formula. The administration of shortacting inhaled bronchodilator was discontinued 6 hours before the examinations of pulmonary functions and the administration of long-acting inhaled bronchodilator was discontinued 12 hours before the examination. The pulmonary functional indexes of FEV1, FVC, and FEV1/FVC were measured three times in all patients to select the optimum value. The patients were subjected to a pulmonary function test at an interval of 15 minutes before and after inhalation of salbutamol aerosol $400 \mu \mathrm{g}$ (Novartis Pharmacy Co., Ltd., Oslo, Switzerland). According to the GOLD/ATS standard, FEV1/FVC $<70 \%$ and FEV $1<80 \%$ after inhalation of bronchodilator. The predicted value was defined as incompletely reversible airway limitation. The COPD severity of the patients definitely diagnosed with COPD was rated on the basis of the decrease of the predicted value of FEV $1 \%$. Class I: FEV $1>80 \%$ predicted value; Class II: $50 \%<\mathrm{FEV} 1<80 \%$ predicted value; Class III: $30 \%<$ FEV $1<50 \%$ predicated value; Class IV: FEV $1<30 \%$ predicted value or FEV $1<50 \%$ predicted value, complicated by respiratory failure. The result of the bronchial dilation test or the provocative test was negative. (the criteria for a positive result of the bronchial dilation test: FEV1 increased by $12 \%$ and FEV1 increased by $>200$ mlafter inhabitation of $400 \mu \mathrm{g}$ of salbutamol aerosol.)

\subsection{Determination of Bone Mineral Density}

The DelphiTM dual energy X-ray rapid systemic bone mineral density tester (DEXA) (Hologic liHologic Inc, Waltham, Massachusetls, USA) was used. The bone mineral density of the anteroposterior lumbar vertebra L1-4 and bilateral femoral neck was determined in all subjects. The apparatus was subject to a vertebral model test on each working day. The test result Area measurement variable coefficient (CV) was below $0.52 \%$ and BMD measurement $\mathrm{CV}$ is below $0.40 \%$. Based on the diagnostic criteria for osteoporosis of WHO, the diagnosis was made on the basis of the $\mathrm{T}$ value. The peak bone mass values of the tested sites of lumbar vertebra L1-4 and femoral neck in patients and those in healthy people of the same gender and race were compared. (1) normal bone mass: less than 1 standard deviation, $\mathrm{T}$ value $\geq 1.0 ;$ (2) osteopenia: any tested site $2.5<\mathrm{T}$ value $<-1.0$; (3) osteoporosis: The $\mathrm{T}$ value at any tested site $\leq-2.5$. According to the standard, based on the actual testing results, the patients were divided into 3 groups: COPD normal bone density group (30 cases); COPD decreased bone density group (30 cases); COPD osteoporosis group.

\subsection{Detection of Cytokines}

$10 \mathrm{ml}$ of fasting elbow venous blood was drawn in the 90 patients in the morning of the next day and centrifuged for 5 minutes at 1000 RPM. The supernatant was collected. The ELISA method (Bio-Rad, Munich, Germany) was used to detect the levels of MMP-9, TIMP-1, and TNF- $\alpha$. The standard was diluted. $100 \mu \mathrm{l}$ of the standard and sample was added to each well. It was incubated for $2 \mathrm{~h}$ at $37^{\circ} \mathrm{C}$. The liquid was discarded and the microplate was not washed. The second antibody was diluted with the diluent as per a proportion of $1: 100.100 \mu$ of the diluted secondary antibody was added to each well. It was incubated for $1 \mathrm{~h}$ at $37^{\circ} \mathrm{C}$. The microplate was washed 3 times. The enzyme-labeled conjugate was added. $90 \mu \mathrm{l}$ of TMB substrate developing solution was added to each well. It was incubated for 10-30 minutes at $37^{\circ} \mathrm{C}$ until the color of the standard exhibited a significant gradient. $50 \mu \mathrm{l}$ of the stop solution was added to each well. It was detected at a wave length of $450 \mathrm{~nm}$ (Thermo Scientific, Waltham, MA).

\subsection{Statistical Method}

The SPSS19.0(SPSS Inc, Chicago, USA) statistical software was used for data analysis. The measurement data were expressed with mean \pm standard deviation. The normal test analysis used the Shapiro-Wilk test. The comparison of the measurement data of normal distribution among groups used the one-way analysis of variance (one-way ANOVA). 
The analysis of the correlation among various parameters used the Pearson analysis. The bone density of lumbar vertebra and bone density of femoral neck served as dependent variables and the levels of serum MMP-9/TIMP-1, TNF- $\alpha$, OPG/RANK/RANKL served as independent variables for multivariate step-wise linear regression analysis. The difference was considered statistically significant when $\mathrm{P}<0.05$.

\section{Result}

\subsection{Comparison of General Clinical Data and Measurements of Bone Mineral Density}

The patients were divided into COPD normal bone mass group, low bone mass group, and osteoporosis group of 30 each according to the bone mineral density result. Pairwise comparisons were made in age, BMI, smoking index, FEV1/FVC, percentage of FEV1 in the predicted value, and CAT scores. There were significant differences in BMI and
CAT scores between the osteoporosis group, the normal bone mass group, and the low bone mass group $(\mathrm{P}<0.01)$. No definite statistical differences were found in age, smoking index, FEV1/FVC, and FEV1\% Pre $(\mathrm{P}>0.05)$. The bone density of lumbar vertebra in the low bone mass group and the osteoporosis group decreased when compared with that in the normal bone mass group and the differences were statistically significant $(\mathrm{P}<0.05, \mathrm{P}<0.01)$. The femoral neck density in the low bone mass group and the osteoporosis group decreased significantly when compared with that in the normal bone mass group and there were significant differences $(\mathrm{P}<0.01)$. The Pearson correlation analysis indicated that The bone density of lumbar vertebra and the bone density of femoral neck were significantly and positively correlated with $\mathrm{BMI}(\mathrm{r}=0.245, \mathrm{P}<0.01 ; \mathrm{r}=0.277$, $\mathrm{P}<0.01$ ). The bone density of lumbar vertebra and the bone density of femoral neck were significantly and negatively correlated with the CAT scores $(\mathrm{F}=-0.356, \mathrm{P}<0.01 ; \mathrm{F}=-0.498$, $\mathrm{P}<0.01)$.

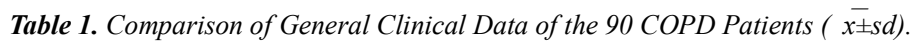

\begin{tabular}{|c|c|c|c|c|c|}
\hline & normal bone mass group $(n=30)$ & low bone mass group $(n=30)$ & osteoporosis group $(n=30)$ & $\mathbf{F}$ & $\mathbf{P}$ \\
\hline age & $64.10 \pm 9.38$ & $65.43 \pm 11.45$ & $65.57 \pm 10.68$ & 1.243 & 0.259 \\
\hline FEV1/FVC & $55.38 \pm 6.45$ & $55.13 \pm 7.59$ & $53.22 \pm 5.65$ & 1.769 & 0.155 \\
\hline FEV1\%Pre & $54.32 \pm 7.49$ & $56.18 \pm 7.55$ & $51.38 \pm 6.26$ & 1.557 & 0.243 \\
\hline smoking index (packets/year) & $31.48 \pm 6.55$ & $32.16 \pm 6.49$ & $33.56 \pm 7.28$ & 1.438 & 0.157 \\
\hline BMI & $26.32 \pm 6.19$ & $23.08 \pm 3.13 * *$ & $20.43 \pm 2.88 * * \wedge \wedge$ & 16.793 & 0.000 \\
\hline CAT scores & $14.49 \pm 4.15$ & $19.66 \pm 5.17 * *$ & $24.57 \pm 5.19 * * \wedge \wedge$ & 27.349 & 0.000 \\
\hline BMD lumbar vertebra & $1.05 \pm 0.18$ & $0.89 \pm 0.42 *$ & $0.65 \pm 0.17 * * \wedge$ & 9.845 & 0.000 \\
\hline BMD femoral neck & $0.87 \pm 0.16$ & $0.76 \pm 0.14 * *$ & $0.58 \pm 0.10 * * \wedge \wedge$ & 31.467 & 0.000 \\
\hline
\end{tabular}

Legend: A comparison with the normal bone mass group indicated $* \mathrm{p}<0.05, * * \mathrm{p}<0.01$; a comparison with the low bone mass group indicated $\wedge \mathrm{p}<0.05$, $\wedge \wedge \mathrm{p}<0.01$

\subsection{Comparison of the Levels of Serum MMP-9/TIMP-1, TNF-a, OPG/RANK/RANKL, SBAP, sOC, and sCTX in the COPD Stable Phase}

There were significant differences in the level of serum MMP-9 among the three groups $(\mathrm{P}<0.01)$. The level of serum MMP-9 was increasing in sequence in the COPD normal bone mass group, low bone mass group, and osteoporosis group and the increase was most significant in the osteoporosis group. The level of serum TIMP-1 was increasing in sequence in the COPD normal bone mass group, low bone mass group, and osteoporosis group and the differences were not statistically significant $(\mathrm{P}>0.05)$. MMP9/TIMP-1 was increasing in sequence in the COPD normal bone mass group, low bone mass group, and osteoporosis group. There were significant differences between the osteoporosis group and the normal bone mass group $(\mathrm{P}<0.01)$. There were significant differences in the serum TNF-A among the three groups $(\mathrm{P}<0.05)$. The level of serum TNF-A was increasing in sequence in the COPD normal bone mass group, the low bone mass group, and the osteoporosis group and the increase was most significant in the osteoporosis group. The level of serum OPG in the COPD osteoporosis group increased when compared with that in the normal bone mass group and there were significant differences $(\mathrm{P}<0.01)$. There were no significant differences in the level of serum OPG between the COPD osteoporosis group and the low bone mass group, and the low bone mass group and the normal bone mass group $(\mathrm{P}>0.05)$. There were no significant differences in the RANK level among the three groups $(\mathrm{P}>0.05)$. The level of serum RANKL in the COPD osteoporosis group was significantly higher than that in the low bone mass group and the normal bone mass group $(\mathrm{P}<0.01)$.

There were no significant differences in RANKL level between the low bone mass group and the normal bone mass group $(\mathrm{P}>0.05)$. The $\mathrm{RANKL} / \mathrm{OPG}$ ratio in the COPD osteoporosis group was significantly higher than that in the low bone mass group and the normal bone mass group $(\mathrm{P}<0.01)$ whereas no significant differences were found between the low bone mass group and the normal bone mass group $(\mathrm{P}>0.05)$. See Table 2 for other indexes. 


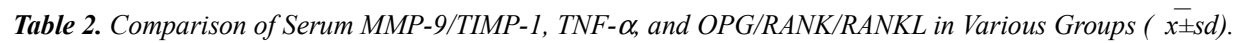

\begin{tabular}{|c|c|c|c|c|c|}
\hline & normal bone mass group $(n=30)$ & low bone mass group $(n=30)$ & osteoporosis group $(n=30)$ & $\mathbf{F}$ & $\mathbf{P}$ \\
\hline MMP-9 (ng/ml) & $32.45 \pm 7.17$ & $48.43 \pm 6.19$ & $83.71 \pm 6.19$ & 488.37 & 0.000 \\
\hline TIMP-1 (ng/ml) & $173.46 \pm 26.88$ & $183.45 \pm 51.54$ & $195.46 \pm 46.66$ & 1.943 & 0.148 \\
\hline MMP-9 and TIMP-1 & $0.17 \pm 0.05$ & $0.31 \pm 0.12$ & $0.46 \pm 0.13$ & 51.336 & 0.000 \\
\hline TNF- $\alpha(\mathrm{ng} / \mathrm{ml})$ & $31.43 \pm 11.16$ & $34.34 \pm 6.29$ & $41.33 \pm 5.23$ & 11.673 & 0.000 \\
\hline $\mathrm{OPG}(\mathrm{pg} / \mathrm{ml})$ & $243.56 \pm 35.79$ & $253.23 \pm 23.56$ & $267.45 \pm 33.43$ & 4.446 & 0.013 \\
\hline RANK $(\mathrm{pg} / \mathrm{ml})$ & $75.58 \pm 11.32$ & $74.67 \pm 7.88$ & $71.24 \pm 8.76$ & 1.557 & 0.221 \\
\hline RANKL (pg/ml) & $494.58 \pm 56.67$ & $517.29 \pm 77.34$ & $615.67 \pm 36.21$ & 34.069 & 0.000 \\
\hline RANKL/OPG & $2.18 \pm 0.35$ & $2.17 \pm 0.26$ & $2.55 \pm 0.26$ & 5.468 & 0.006 \\
\hline $\operatorname{sBAP}(\mu / 1)$ & $65.34 \pm 5.56$ & $41.55 \pm 5.08^{* *}$ & $36.45 \pm 8.55^{* *}$ & 155.145 & 0.000 \\
\hline $\mathrm{sOC}(\mu \mathrm{g} / \mathrm{l})$ & $42.57 \pm 6.23$ & $32.43 \pm 4.44^{* *}$ & $31.45 \pm 4.68^{* *}$ & 28.436 & 0.000 \\
\hline $\mathrm{sCTX}(\mu \mathrm{g} / \mathrm{l})$ & $0.46 \pm 0.04$ & $0.48 \pm 0.16$ & $0.55 \pm 0.03 * * \wedge \wedge$ & 153.548 & 0.000 \\
\hline
\end{tabular}

Legend: A comparison with the normal bone mass group indicated $* \mathrm{p}<0.05,{ }^{*} \mathrm{p}<0.01$. A comparison with the low bone mass group indicated $\wedge \mathrm{p}<0.05$, $\wedge \mathrm{p}<0.01$.

\subsection{Correlation Analysis of Various Parameters}

The Pearson correlation analysis for relevant indexes indicated that the bone density of lumbar vertebra and bone density of femoral neck, and the serum MMP-9 were significantly and negatively correlated $(\mathrm{r}=-0.445, \mathrm{P}<0.01)$ and $(\mathrm{r}=-0.659, \mathrm{P}<0.01)$. The bone density of lumbar vertebra, bone density of femoral neck, and RANKL $(\mathrm{F}=0.458, \mathrm{P}<0.01)$, and $(\mathrm{r}=-0.557, \mathrm{P}<0.01), \mathrm{RANKL} / \mathrm{OPG}$ ratio $(\mathrm{r}=-0.337, \mathrm{P}<0.01$ and $\mathrm{F}=-0.467, \mathrm{P}<0.01)$ were significantly and negatively correlated. FEV1\%Pre and the serum MMP-9 were significantly and negatively correlated $(\mathrm{F}=-0.237, \mathrm{P}<0.05)$. FEVI\%Pre and RANKL $(\mathrm{r}=-0.336$, $\mathrm{P}<0.01)$, RANKL/OPG ratio $(\mathrm{r}=-0.465, \mathrm{P}<0.05)$ The bone density of lumbar vertebra and bone density of femoral neck, and the serum MMP-9 were significantly and negatively correlated $(\mathrm{F}=-0.455, \mathrm{P}<0.01 ; \mathrm{r}=-0.655, \mathrm{P}<0.01)$. The bone density of lumbar vertebra, and bone density of femoral neck, and the sBAP were significantly and positively correlated $(\mathrm{r}=0.423, \mathrm{P}<0.01 ; \mathrm{r}=0.718, \mathrm{P}<0.01)$. The bone density of lumbar vertebra, and bone density of femoral neck, and the sOC were significantly and positively correlated $(\mathrm{F}=$ $0.367, \mathrm{P}<0.01 ; \mathrm{r}=0.559, \mathrm{P}<0.01)$. The bone density of lumbar vertebra, and bone density of femoral neck, and the SCTX were significantly and positively correlated $(\mathrm{r}=$ $0.456, \mathrm{P}<0.01 ; \mathrm{F}=0.654, \mathrm{P}<0.01)$. The FEVI\%Pre and the serum MMP-9 were significantly and positively correlated $(\mathrm{r}$ $=-0.227, \mathrm{P}<0.05)$. FEVI\%Pre and sBAP, and sOC were significantly and positively correlated $(\mathrm{F}=0.238, \mathrm{P}<0.01 ; \mathrm{r}=$ $0.245, \mathrm{P}<0.01)$. FEVI\%Pre and SCTX were significantly and negatively correlated $(\mathrm{r}=-0.238, \mathrm{P}<0.05)$.

\section{Discussion}

Chronic obstructive pulmonary disease (COPD) is a common chronic respiratory disease characterized by airway limitation with a high disability rate and case fatality rate. With the progress of the medical conditions, the survival rate of the COPD patients is improving. As two important complications of COPD, osteoporosis and fragility fractures have been arousing more and more attention. The morbidity of osteoporosis in the COPD patients is higher than that in the normal people. The vital capacity and total lung capacity of the patients with severe osteoporosis are decreasing with the increase of thoracic vertebrae fractures. Osteoporosis fractures have been one of the factors that severely decrease the COPD patients' quality of life [21]. The pathogenesis of osteoporosis secondary to COPD still remain unclear.

Osteoporosis secondary to COPD results from multiple factors from the etiology perspective. Many previous studies have proposed multiple etiological factors related to COPD complicated by osteoporosis, such as BMI, age, declined quality of life, decrease of FEV1, smoking, and administration of glucocorticoid etc. [22, 23]. Some researchers have pointed out that only the relationship between BMI and osteoporosis is generally accepted while the roles of other factors are still controversial. In the research, there are significant differences in BMI and CAT scores between the group of stable phase COPD complicated by osteoporosis, and the normal bone mass group and the low bone mass group. No definite statistical differences are found in age, smoking indexes, FEV1/FVC, and FEV1\%Pre. BMI in the COPD osteoporosis group is lower than that in the group of decreased COPD bone mineral density and the group of normal COPD bone mineral density. BMI in the group of decreased COPD bone mineral density is lower than that in the group of normal COPD bone mineral density. Their differences are statistically significant. In the research, the bone density of lumbar vertebra and bone density of femoral neck and BMI are significantly and positively correlated. The results are consistent with many previous research results.

Both BMI and age are the factors definitely associated with the patients' quality of life [24]. Our research result indicates that the CAT score in the COPD osteoporosis group are higher than that in the group of decreased COPD bone mineral density and the group of normal COPD bone mineral density. The CAT score in the group of decreased COPD bone mineral density is higher than that in the group of normal COPD bone mineral density. All of these differences are statistically significant. The bone density of lumbar vertebra and the bone density of femoral neck and the CAT scores are significantly and negatively correlated and the correlation is statistically significant. The above result shows that the lower the patients' bone mineral density, the poorer their quality of life will be. FEV1\%Pre and the bone density of lumbar vertebra and the bone density of femoral neck are 
significantly and positively correlated. However, some research has not found the correlation between the decrease of bone mineral density and the airflow obstruction [25]. In conclusion, there are various factors related to osteoporosis secondary to COPD. It may result from the interactions among multiple factors. More in-depth research of larger sample size is expected to demonstrate this result.

The research finds that there are significant differences in serum MMP-9 among the three groups. The level of serum MMP-9 is increasing in sequence in the COPD normal bone mass group, the decreased bone mass group, and the osteoporosis group. The increase is most significant in the osteoporosis group. The level of serum TIMP-1 is increasing in sequence in the COPD normal bone mass group, the low bone mass group, and the osteoporosis group but these differences are not statistically significant. The bone density of lumbar vertebra, bone density of femoral neck and FEVI\%Pre, and the serum MMP-9 are significantly and negatively correlated. The correlation between the bone density of femoral neck and MMP-9 is better than the correlation between the bone density of lumbar vertebra and MMP-9. MMP-9/TIMP-1 is increasing in sequence in the COPD normal bone mass group, the decreased bone mass group, and the osteoporosis group. There are significant differences in MMP-9/TIMP-1 between the osteoporosis group, and the decreased bone mass group and the normal bone mass group. When osteoporosis occurs, the osteoclasts will be activated and the MMP-9 is highly expressed thus promoting bone resorption. TIMP-1 produced by osteoblasts do not increase and the MMP-9/TIMP-1 ratio is unbalanced. The activity of osteoclasts increases while the activity of osteoblasts decreases relatively. The rate of bone formation decreases and irreversible bone loss occurs thus leading to osteoporosis. Unbalance of MMP-9/TIMP-1 ratio may be one of the important causes of COPD complicated by osteoporosis.

The roles of inflammatory mediators have been arousing more and more attention during COPD onset. TNF- $\alpha$ is one of the inflammatory factors closely associated with incidence and progression of COPD [26]. The level of serum inflammatory factor TNF- $\alpha$ in the group of patients with COPD complicated by osteoporosis is significantly higher than that in the group of normal bone mass and the group of decreased bone mass and the differences are statistically significant. MMP-9 and TNF- $\alpha$ are significantly and positively correlated. The research finds that the levels of inflammatory factors of IL- 6 and TNF-a will increase in injury diseases and decrease in the recovery. The inflammatory factors can also directly act upon the osteoclasts and their precursor cells, lead to increased activity of the osteoclasts, and cause and accelerate bone destruction and loss of bone mass. In addition to this, these inflammatory factors can also interact with the protein OPG/RANK/RANKL systems related to osteoporosis and jointly regulate the bone metabolism. It is inferred that the damage to pulmonary parenchyma and the damage to the bone structure may be correlated but the potential mechanism still remains unclear. The mechanism may be associated with the action of systemic inflammatory factors on both the pulmonary parenchyma and the bone matrix.

OPG/RANKL/RANK are new markers. The bone density of lumbar vertebra, bone density of femoral neck, and the RANKL, RANKL/OPG ratio are significantly and negatively correlated in the COPD patients [27]. The bone density of lumbar vertebra and bone density of femoral neck, and other cytokines (TIMP-1, TNF-a, OPG, and RANK) are not significantly correlated. The FEV1\%Pre, and the RANKL, and RANKL/OPG ratio are significantly and negatively correlated. The FEV1\%Pre and other cytokines (TIMP-1, TNF- $\alpha$, OPG, and RANK) are not significantly correlated. The level of serum OPG in the patients with COPD complicated by osteopenia/osteoporosis is significantly higher than that in the patients without decreased bone mineral density. The level of serum OPG and the bone mineral density of hip bone are negatively correlated. However, the research involves no the level of serum RANKL and RANKL/OPG ratio. In our research, the serum OPG and the RANKL/OPG ratio in the osteoporosis group is higher than that in the normal bone mass group while no significant differences are found between the decreased bone mass group and the normal bone mass group.

\section{Conclusion}

The damage to pulmonary parenchyma and the damage to bone structure may be correlated but the potential mechanism still remains unclear. The mechanism may be associated with the action of systemic inflammatory factors on both the pulmonary parenchyma and the bone matrix. Osteoporosis is one of the important systemic complications of COPD and results from various factors. The inflammatory cells of the COPD patients will release more MMP-9 which can be involved in blood circulation and a larger number of inflammatory factors such as TNF- $\alpha$ etc. due to airway inflammation. These inflammatory factors act on both the alveolar extracellular matrix and the bone matrix thus leading to unbalance of OPG/RANK/RANKL, increased RANKL/OPG ratio, activation of osteoclasts, high expression of MMP-9, increased absorption of bone matrix, and increased likelihood of osteoporosis. MMP-9 released by the osteoclasts will exacerbate the unbalance of the pulmonary MMP9/TIMP-1 ratio, increase the degradation of the alveolar extracellular matrix of the alveolar wall, aggravate pulmonary structural damage and emphysema. MMPs participate in the pathophysiologic process of osteoporosis secondary to COPD as an important factor. The MMP-9/TIMP-1, TNF- $\alpha$, OPG/RANK/RANKL systems may play a synergistic effect in incidence and progression of pulmonary parenchyma damage and osteoporosis. In-depth research on the relationships between cytokines and the OPG/RANK/RANKL systems can provide a better and newer method for prevention and treatment of COPD complicated by osteoporosis.

\section{Acknowledgements}

The present study was funded by the following projects: 
The Nature Science Foundation of Shandong (Grant no. ZR2014HQ060).

\section{References}

[1] Yang L, He Q. [Effects of inhaled corticosteroids on osteoporosis in patients with chronic obstructive pulmonary disease]. Zhonghua Jie He He Hu Xi Za Zhi 2015; 38: 777-779.

[2] Brinchault G, Diot P, Dixmier A, Goupil F, Guillais P, GutGobert C, Leroyer C, Marchand-Adam S, Meurice JC, Morel $\mathrm{H}$, Person C, Cavailles A. [Comorbidities of COPD]. Rev Pneumol Clin 2015; 71: 342-349.

[3] Terasaki J, Singh G, Zhang W, Wagner P, Sharma G. Using EMR to improve compliance with clinical practice guidelines for management of stable COPD. Respir Med 2015; 109: 1423-1429.

[4] Takahashi S, Betsuyaku T. The chronic obstructive pulmonary disease comorbidity spectrum in Japan differs from that in western countries. Respir Investig 2015; 53: 259-270.

[5] Kooman JP, Shiels PG, Stenvinkel P. Premature aging in chronic kidney disease and chronic obstructive pulmonary disease: similarities and differences. Curr Opin Clin Nutr Metab Care 2015; 18: 528-534.

[6] Ajmera M, Sambamoorthi U, Metzger A, Dwibedi N, Rust G, Tworek C. Multimorbidity and COPD Medication Receipt Among Medicaid Beneficiaries With Newly Diagnosed COPD. Respir Care 2015; 60: 1592-1602.

[7] Liu WT, Kuo HP, Liao TH, Chiang LL, Chen LF, Hsu MF, Chuang HC, Lee KY, Huang CD, Ho SC. Low bone mineral density in COPD patients with osteoporosis is related to low daily physical activity and high COPD assessment test scores. Int J Chron Obstruct Pulmon Dis 2015; 10: 1737-1744.

[8] Durup D, Jorgensen HL, Christensen J, Tjonneland A, Olsen A, Halkjaer J, Lind B, Heegaard AM, Schwarz P. A Reverse JShaped Association Between Serum 25-Hydroxyvitamin D and Cardiovascular Disease Mortality: The CopD Study. J Clin Endocrinol Metab 2015; 100: 2339-2346.

[9] Jorgensen TS, Hansen AH, Sahlberg M, Gislason GH, TorpPedersen C, Andersson C, Holm E. Nationwide time trends and risk factors for in-hospital falls-related major injuries. Int J Clin Pract 2015; 69: 703-709.

[10] Corsonello A, Scarlata S, Pedone C, Bustacchini S, Fusco S, Zito A, Incalzi RA. Treating COPD in Older and Oldest Old Patients. Curr Pharm Des 2015; 21: 1672-1689.

[11] Chen SJ, Liao WC, Huang KH, Lin CL, Tsai WC, Kung PT, Chang $\mathrm{KH}$, Kao $\mathrm{CH}$. Chronic obstructive pulmonary disease and allied conditions is a strong independent risk factor for osteoporosis and pathologic fractures: a population-based cohort study. QJM 2015; 108: 633-640.

[12] Pandrea I, Landay A, Wilson C, Stock J, Tracy R, Apetrei C. Using the pathogenic and nonpathogenic nonhuman primate model for studying non-AIDS comorbidities. Curr HIV/AIDS Rep 2015; 12: 54-67.

[13] Chubachi S, Nakamura H, Sasaki M, Haraguchi M, Miyazaki M, Takahashi S, Tanaka K, Funatsu Y, Asano K, Betsuyaku T. Polymorphism of LRP5 gene and emphysema severity are associated with osteoporosis in Japanese patients with or at risk for COPD. Respirology 2015; 20: 286-295.
[14] Bousquet J, Anto JM, Berkouk K, Gergen P, Antunes JP, Auge P, Camuzat T, Bringer J, Mercier J, Best N, Bourret R, Akdis M, Arshad SH, Bedbrook A, Berr C, Bush A, Cavalli G, Charles MA, Clavel-Chapelon F, Gillman M, Gold DR, Goldberg M, Holloway JW, Iozzo P, Jacquemin S, Jeandel C, Kauffmann F, Keil T, Koppelman GH, Krauss-Etschmann S, Kuh D, Lehmann S, Carlsen KC, Maier D, Mechali M, Melen E, Moatti JP, Momas I, Nerin P, Postma DS, Ritchie K, Robine JM, Samolinski B, Siroux V, Slagboom PE, Smit HA, Sunyer J, Valenta R, Van de Perre P, Verdier JM, Vrijheid M, Wickman M, Yiallouros P, Zins M. Developmental determinants in non-communicable chronic diseases and ageing. Thorax 2015; 70: 595-597.

[15] Matera MG, Cardaci V, Cazzola M, Rogliani P. Safety of inhaled corticosteroids for treating chronic obstructive pulmonary disease. Expert Opin Drug Saf 2015; 14: 533-541.

[16] McGarvey L, Lee AJ, Roberts J, Gruffydd-Jones K, McKnight E, Haughney J. Characterisation of the frequent exacerbator phenotype in COPD patients in a large UK primary care population. Respir Med 2015; 109: 228-237.

[17] Chun P. Role of sirtuins in chronic obstructive pulmonary disease. Arch Pharm Res 2015; 38: 1-10.

[18] Malinovschi A, Masoero M, Bellocchia M, Ciuffreda A, Solidoro P, Mattei A, Mercante L, Heffler E, Rolla G, Bucca C. Severe vitamin D deficiency is associated with frequent exacerbations and hospitalization in COPD patients. Respir Res 2014; 15: 131.

[19] Watanabe R, Tanaka T, Aita K, Hagiya M, Homma T, Yokosuka K, Yamakawa H, Yarita T, Tai N, Hirano J, Inoue D, Okazaki R. Osteoporosis is highly prevalent in Japanese males with chronic obstructive pulmonary disease and is associated with deteriorated pulmonary function. J Bone Miner Metab 2015; 33: 392-400.

[20] Park SJ, Foreman MG, Demeo DL, Bhatt SP, Hansel NN, Wise RA, Soler X, Bowler RP. Menthol cigarette smoking in the COPDGene cohort: relationship with COPD, comorbidities and CT metrics. Respirology 2015; 20: 108-114.

[21] Malik RD, Lapin B, Wang CE, Lakeman JC, Helfand BT. Are we testing appropriately for low testosterone? Characterization of tested men and compliance with current guidelines. J Sex Med 2015; 12: 66-75.

[22] Lin YH, Tsai CL, Chien LN, Chiou HY, Jeng C. Newly diagnosed gastroesophageal reflux disease increased the risk of acute exacerbation of chronic obstructive pulmonary disease during the first year following diagnosis--a nationwide population-based cohort study. Int J Clin Pract 2015; 69: 350-357.

[23] A VR. Inhalational Steroids and Iatrogenic Cushing's Syndrome. Open Respir Med J 2014; 8: 74-84.

[24] Khow KS, Yong TY. Atypical femoral fracture in a patient treated with denosumab. J Bone Miner Metab 2015; 33: 355-358.

[25] Chee C, Sellahewa L, Pappachan JM. Inhaled corticosteroids and bone health. Open Respir Med J 2014; 8: 85-92.

[26] Aryal S, Diaz-Guzman E, Mannino DM. Influence of sex on chronic obstructive pulmonary disease risk and treatment outcomes. Int J Chron Obstruct Pulmon Dis 2014; 9: 1145-1154.

[27] Escamilla R. [COPD: beyond the respiratory system]. Presse Med 2014; 43: 1381-1386. 